\title{
ABSOLUTE ABEL SUMMABILITY AND CAPACITY
}

\author{
G. V. WELLAND
}

Abstract. Precise limits on the size of exceptional sets for which functions in the Lebesgue class, $\mathcal{L}_{\alpha}^{p}$, can fail to be absolutely Abel summable are given in terms of Bessel capacity.

1. Introduction. In this note we present two theorems which generalize results of [1] and [2]. We do this in the context of the Lebesgue classes $\mathcal{L}_{\alpha}^{p}$ and the capacity theory developed in [3]. Here we introduce those facts from [3] which are for the most part contained in Theorem 16 of that paper.

Let $Q_{k}=\left\{x \in E_{k}:-\frac{1}{2}<x_{i}<\frac{1}{2}, i=1,2, \ldots, k\right\}$ and $k>2$ be the torus in $k$-dimensional Euclidean space. Let $0<\alpha<k$, and $g_{\alpha}$ be the kernel of the Bessel potential which is given by the positive function whose Fourier coefficients are $\hat{g}_{\alpha}(n)=\left(1+4 \pi^{2}|n|^{2}\right)^{-\alpha / 2}$ where $n$ is a point in the $k$-dimensional integral lattice plane. Let $\mathrm{e}_{1}^{+}$be the space of all nonnegative Radon measures of finite total variation on $Q_{k}$. If $\nu \in \mathfrak{L}_{1}^{+},\|\nu\|$ denotes its total variation. For $\nu \in \mathcal{E}_{1}^{+}, g_{\alpha}(\nu, x)=\left(g_{\alpha} * \nu\right)(x)$. For $Z \subset Q_{k}$ an analytic set the capacity of $Z$ is defined for $1<p<\infty$ by

$$
c_{\alpha, p}(Z)=\sup \|\nu\|,
$$

where the supremum is taken over all those $\nu \in \mathcal{L}_{1}^{+}$concentrated on $Z$ for which $\left\|g_{\alpha}(\nu, \cdot)\right\|_{p^{\prime}}<1$ and $p^{\prime}=p /(p-1)$.

If $Z$ has positive capacity then there is a nontrivial $\mu \in \mathcal{L}_{1}^{+}$satisfying the variational problem of (1.1), the function $f$ defined by

$$
f^{p-1}(x)=\left(c_{\alpha, p}(Z)\right)^{p-1} g_{\alpha}(\mu, x)
$$

is in $L^{p}$, and $\|f\|_{p}=c_{\alpha, p}(Z)$. Moreover $\mu$ is concentrated on the set $Z \cap\{x$ : $\left.\left(f * g_{\alpha}\right)(x)=1\right\}=Z_{0}$ and the set $Z-Z_{0}$ has zero $c_{\alpha, p}$ capacity. Such $f$ and $\mu$ are called capacitary distributions for $Z$. Let $f_{0}=f /\|f\|_{p}$ and $\mu_{0}=\mu /\|\mu\|$. Finally, for any function $h$ satisfying $\left(h * g_{\alpha}\right)(x) \geqslant 1$ on $Z$ and $\|h\|_{p}=1$, and any measure $\nu$ concentrated on $Z$ satisfying $\|\nu\|=1$, and $\left\|g_{\alpha}(\nu, \cdot)\right\|_{p^{\prime}}<1$ we have

$$
g_{\alpha}\left(\mu_{0}, h\right)<g_{\alpha}\left(\mu_{0}, f_{0}\right)<g_{\alpha}\left(\nu, f_{0}\right)
$$

where $g_{\alpha}(\mu, f)=\iint f(y) g_{\alpha}(x-y) d \mu(x) d y$.

We say a function $f$ belongs to the Lebesgue class $\mathcal{L}_{\alpha}^{p}$ if $f$ can be written as $f_{0} * g_{\alpha}$ for some $f_{0}$ in $L^{p}=L^{p}\left(Q_{k}\right)$.

Received by the editors September 21, 1978.

AMS (MOS) subject classifications (1970). Primary 42A92, 42A16.

(C) 1979 American Mathematical Society 0002-9939/79/0000-0512/\$02.50 
2. Absolute Abel summability. In this section we deal with absolute Abel summability of multiple Fourier series. We say the Fourier series of a function $f$ is absolutely Abel summable at a point $x^{0}$ if

$$
\int_{0}^{1}\left|\frac{\partial f}{\partial t}\left(x^{0}, t\right)\right| d t<\infty
$$

where

$$
f(x, t)=\sum_{m} \hat{f}(m) e^{2 \pi(i m \cdot x-|m| t)} \quad \text { for } t>0 .
$$

The theorems we prove are the following.

TheOREM 1. Let $f$ be a function of class $\mathcal{L}_{\alpha}^{p}\left(Q_{k}\right)$. Then $f$ is absolutely Abel summable except possibly on a set of zero $c_{\alpha, p}$ capacity.

THEOREM 2. Let $Z$ be a closed set in $Q_{k}$ which is of zero $c_{\alpha, p}$ capacity. Then there exists a function $f$ in $\mathcal{L}_{\alpha}^{p}\left(Q_{k}\right)$ such that

$$
\int_{0}^{1}\left|\frac{\partial f(x, t)}{\partial t}\right| d t=\infty \quad \text { for each } x \in Z
$$

It should be noted that in [2] these theorems are given for the case $p=2$ and ordinary capacity, which is motivated by one dimensional results of [1].

Let

$$
g_{\alpha}(x, t)=\sum_{m} \frac{e^{2 \pi i m \cdot x-2 \pi t|m|}}{\left(1+4 \pi^{2}|m|^{2}\right)^{\alpha / 2}}, \quad t>0 .
$$

We have the following which can be obtained from an application of the Poisson summation formula and the properties of Bessel potentials listed in 87 of [3].

(2.1) For $\alpha>0, g_{\alpha}(x, t) \rightarrow g_{\alpha}(x)$ provided $x$ is not a lattice point, as $t \rightarrow 0$.

(2.2) The function $g_{\alpha}(x)$ is continuous if $x$ is not a lattice point, $g_{\alpha} \in L^{1}$ and $g_{\alpha}>0$.

$$
\int_{0}^{1}\left|\frac{\partial g_{\alpha}}{\partial t}(x, t)\right| d t<g_{\alpha}(x)+c(k) \quad \text { where } c(k)=\sum_{m} e^{-2 \pi|m|} .
$$

To see that (2.3) holds, note that

$$
\int_{0}^{1}\left|\frac{\partial g_{\alpha}(x, t)}{\partial t}\right| d t<\lim _{\varepsilon \rightarrow 0} \int_{e}^{1}\left|\frac{\partial g_{\alpha}(x, t)}{\partial t}\right| d t
$$

and begin by applying the Poisson summation formula to justify that

$$
\begin{aligned}
& \frac{\partial g_{\alpha}(x, t)}{\partial t}=\int_{y \in \mathbf{R}^{k}} g_{\alpha}(x-y) \frac{\partial P_{t}}{\partial t}(y) d y \\
& \text { where } P_{t}(y)=C \frac{t}{\left(t^{2}+|y|^{2}\right)^{(k+1) / 2}} .
\end{aligned}
$$


The positivity of $g_{\alpha}$ is used several times in the following. It follows that

$$
\begin{aligned}
\int_{e}^{1}\left|\frac{\partial g_{\alpha}(x, t)}{\partial t}\right| d t & <\int_{\varepsilon}^{1} \int g_{\alpha}(x-y)\left|\frac{\partial P_{t}}{\partial t}(y)\right| d y d t \\
& <\left\{\int_{\varepsilon}^{1} \int_{|y|<2 \sqrt{k}}+\int_{e}^{1} \int_{|y|>2 \sqrt{k}}\right\} g_{\alpha}(x-y)\left|\frac{\partial P_{t}}{\partial t}\right| d y d t \\
& \equiv A+B .
\end{aligned}
$$

Direct calculation of $\partial P_{t} / \partial t$ shows that $\partial P_{t}(y) / \partial t>0$ if and only if $|y|>\sqrt{k} t$. Define

$$
l=l(y, \varepsilon)= \begin{cases}\varepsilon, & |y|<\varepsilon \sqrt{k}, \\ 1, & |y|>\sqrt{k}, \\ |y| / \sqrt{k}, & \varepsilon \sqrt{k}<|y|<\sqrt{k} .\end{cases}
$$

Then it follows that

$$
\begin{aligned}
A= & -\int_{|y|<2 \sqrt{k}} g_{\alpha}(x-y) \int_{\varepsilon}^{l} \frac{\partial P_{t}}{\partial t}(y) d t d y \\
& +\int_{|y|<2 \sqrt{k}} g_{\alpha}(x-y) \int_{l}^{l} \frac{\partial P}{\partial t}(y) d t d y \\
= & \int_{|y|<2 \sqrt{k}} g_{\alpha}(x-y)\left[P_{\varepsilon}(y)-P_{l}(y)\right] d y \\
& +\int_{|y|<2 \sqrt{k}} g_{\alpha}(x-y)\left[P_{1}(y)-P_{l}(y)\right] d y \\
< & \int_{|y|<2 \sqrt{k}} g_{\alpha}(x-y)\left[P_{e}(y)+P_{1}(y)\right] d y \\
< & g_{\alpha}(x, \varepsilon)+\int_{|y|<2 \sqrt{k}} g_{\alpha}(x-y) P_{1}(y) d y .
\end{aligned}
$$

Similar considerations for $\partial P_{t}(y) / \partial t$ lead to

$$
B=\int_{|y|>2 \sqrt{k}} g_{\alpha}(x-y)\left[P_{1}(y)-P_{e}(y)\right] d y<\int_{|y|>2 \sqrt{k}} g_{\alpha}(x-y) P_{1}(y) d y .
$$

Combining the estimates for $A$ and $B$ gives

$$
\int_{0}^{1}\left|\frac{\partial g_{\alpha}(x, t)}{\partial t}\right| d t<\lim _{\varepsilon \rightarrow 0} g_{\alpha}(x, \varepsilon)+g_{\alpha}(x, 1) .
$$

Again by the Poisson summation formula

$$
g_{\alpha}(x, 1)<\left|\sum_{m} \frac{1}{\left(1+4 \pi^{2}|m|^{2}\right)^{\alpha / 2}} e^{-2 \pi|m|} e^{i m \cdot x}\right|<c(k) .
$$


Next, let

$$
\begin{aligned}
f(x, t) & =\sum_{m} \hat{f}(m) e^{2 \pi i m \cdot x-2 \pi|m| t} \\
& =\sum_{m} \hat{f}_{0}(m) \frac{1}{\left(1+4 \pi^{2}|m|^{2}\right)^{\alpha / 2}} e^{2 \pi i m \cdot x-2 \pi|m| t} \\
& =\int_{Q_{k}} f_{0}(y) \cdot g_{\alpha}(x-y, t) d y
\end{aligned}
$$

It follows that

$$
\frac{\partial f}{\partial t}(x, t)=\int_{Q_{k}} f_{0}(y) \frac{\partial g_{\alpha}}{\partial t}(x-y, t) d y
$$

so that

$$
\begin{aligned}
\int_{0}^{1}\left|\frac{\partial f}{\partial t}(x, t)\right| d t & <\int_{Q_{k}}\left|f_{0}(y)\right| \int_{0}^{1}\left|\frac{\partial g_{\alpha}}{\partial t}(x-y, t)\right| d y d t \\
& <c(k) \int_{Q_{k}}\left|f_{0}(y)\right| d y+\left(\left|f_{0}\right| * g_{\alpha}\right)(x) .
\end{aligned}
$$

We now present the proof of Theorem 1 .

Proof of Theorem 1. Let $E_{\infty}=\left\{x: \int_{0}^{1}|\partial f(x, t) / \partial t| d t=\infty\right\}$ and suppose that $E_{\infty}$ has positive $c_{\alpha, p}$ capacity. Then there exists a measure $\mu$ (capacitary distribution) concentrated on $B_{\infty}=\left\{x: g_{\alpha}\left(x, f_{\mu}\right)=1\right\} \cap E_{\infty}$ where $f_{\mu}(y)=$ $c_{\alpha p}\left(E_{\infty}\right)\left[g_{\alpha}(\mu, y)\right]^{1 /(p-1)}$. We use the notation compatible with $\S 1$ :

$$
\begin{aligned}
& g_{\alpha}(\mu, y)=\int_{Q_{k}} g_{\alpha}(x-y) d \mu(x), \\
& g_{\alpha}\left(x, f_{\mu}\right)=\int_{Q_{k}} g_{\alpha}(x-y) f_{\mu}(y) d y, \\
& c_{\alpha, p}\left(E_{\infty}\right)=\int f_{\mu}^{p}(y) d y=c_{\alpha, p}\left(E_{\infty}\right) \int g_{\alpha}(\mu, y)^{p^{\prime}} d y,
\end{aligned}
$$

and $\|\mu\|=c_{\alpha, p}\left(E_{\infty}\right)>0$.

In this case, let $\nu=c_{\alpha p}^{-1}\left(E_{\infty}\right) \mu$ so that $\|\nu\|=1$ and $\nu\left(Q_{k}-E_{\infty}\right)=0$. Then

$$
\begin{aligned}
\iint\left|f_{0}(y)\right| g_{\alpha}(x-y) d \nu(x) d y & =\int\left|f_{0}(y)\right| \cdot g_{\alpha}(\nu, y) d y \\
& <\left\|f_{0}\right\|_{p} \cdot\left\|g_{\alpha}(\nu, \cdot)\right\|_{p^{\prime}}=\left\|f_{0}\right\|_{p} \cdot c_{\alpha p}^{-1}\left(E_{\infty}\right) .
\end{aligned}
$$

The last inequality follows from the fact that $\left\|g_{\alpha}(\mu, \cdot)\right\|_{p^{\prime}}<1$.

We now have that

$$
\int_{Q_{k}} \int_{0}^{1}\left|\frac{\partial f}{\partial t}(x, t)\right| d t d \nu(x)=\infty
$$


while on the other hand, by (2.5) and (2.6), we have

$$
\begin{aligned}
\int_{Q_{k}} \int_{0}^{1} \mid \frac{\partial f}{\partial t} & (x, t) \mid d t d \nu(x) \\
& <c(k)\left|Q_{k}\right|^{1 / p^{\prime}}\left\|f_{0}\right\|_{p}+\int\left|f_{0}\right|(y) g_{\alpha}(x-y) d \nu(x) d y \\
& =\left[c(k)\left|Q_{k}\right|^{1 / p^{\prime}}+c_{\alpha p}^{-1}\left(E_{\infty}\right)\right]\left\|f_{0}\right\|_{p}<\infty .
\end{aligned}
$$

This contradiction completes the proof of Theorem 1.

3. Counterexample. We now give the construction of the counterexample for Theorem 2 .

Let $Z$ be a closed set in $Q_{k}$ such that $c_{\alpha p}(Z)=0$. Since $c_{\alpha, p}$ is a capacity, we can approximate $c_{\alpha, p}(Z)$ by $c_{\alpha, p}\left(K_{\rho}\right)$ where $K_{\rho}=\{x: \operatorname{dist}(x, Z)<\rho\}$; that is,

$$
c_{\alpha, p}(Z)=\lim _{\rho \rightarrow 0} c_{\alpha, p}\left(K_{\rho}\right) \text {. }
$$

For each $\rho>0$ let $\mu_{\rho}$ and $f_{\rho}$ be the capacitary distributions for $K_{\rho}$ with $f_{\rho}(y)^{p-1}=c_{\alpha, p}\left(K_{\rho}\right)^{p-1} g_{\alpha}\left(\mu_{\rho}, y\right)$ almost everywhere and $f_{\rho} * g_{\alpha}(x)=1$ almost everywhere with respect to capacity $c_{\alpha, p}$ on $K_{\rho}$. Then it follows that

$$
\int g_{\alpha}(x-y)\left\{\int g_{\alpha}(y-z) d \mu_{\rho}(z)\right\}^{1 /(p-1)} d y=c_{\alpha p}\left(K_{\rho}\right)^{-1}
$$

almost everywhere in $K_{\rho}$ with respect to $c_{\alpha p}$ capacity. We choose a sequence $\left\{\mu_{\rho_{1}}\right\}$ associated in the above fashion such that $c_{\alpha_{p}}\left(K_{\rho_{1}}\right)=\left\|\mu_{\rho_{1}}\right\| \rightarrow 0$.

Then $\left\|f_{\rho}\right\|_{p}=c_{\alpha, p}\left(K_{\rho}\right)$. Note that $f_{\rho}$ is not constant on $K_{\rho}$ but it has a potential which is equal to one almost everywhere with respect to $c_{\alpha p}$ capacity. Let

$$
f_{0}^{k}=\sum_{j=1}^{k} c_{j} f_{\rho_{j}} \quad \text { and } \quad f^{k}=\sum_{j=1}^{k} g_{\alpha} *\left(c_{j} f_{\rho_{j}}\right)
$$

Hence $f^{k}$ is in $\mathcal{E}_{\alpha}^{p}\left(Q_{k}\right)$. For $c_{j}$ use $j^{-\delta} c_{\alpha, p}\left(K_{\rho_{j}}\right)^{-1}$ with $\delta>1$ so that we have $\left\|f_{0}^{k}\right\|_{p}<\sum_{j=1}^{k} j^{-\delta}<\infty$ and $\left\{f_{0}^{k}\right\}$ forms a Cauchy sequence in $L^{p}\left(Q_{k}\right)$. Let $f_{0}$ denote the limit of $f_{0}^{k}$ as $k$ tends to infinity. Since $f_{\beta}$ is a positive function, $f_{0}$ is also the pointwise limit of $f_{0}^{k}$. Furthermore, for almost every $x$ in $K_{\rho_{k}}$ with respect to $c_{\alpha, p}$ capacity, we have

$$
\left(f_{0}^{k} * g_{\alpha}\right)(x)>\sum_{j=1}^{k} j^{-\delta} c_{\alpha, p}\left(K_{\rho_{j}}\right)^{-1}\left(f_{\rho_{j}} * g_{\alpha}\right)(x)=\sum_{j=1}^{k} j^{-\delta} c_{\alpha, p}\left(K_{\beta_{j}}\right)^{-1} \text {. }
$$

We choose the sequence $\left\{\rho_{j}\right\}$ such that $c_{\alpha, p}\left(K_{\beta}\right)<j^{-\delta}$. Then in $K_{\rho_{k}}$, $\left(P_{t} * f_{0}^{k} * g_{\alpha}\right)$ tends to $\sum_{j=1}^{k} j^{-\delta} c_{\alpha, p}\left(K_{\rho_{j}}\right)^{-1}>k$ as $t$ tends to zero, since $f_{0}^{k} * g_{\alpha}$ is essentially constant in $K_{\rho_{k}}$.

Since

$$
|f(x, 1)-f(x, t)|<\int_{t}^{1}\left|\frac{\partial f}{\partial s}(x, s)\right| d s \text { and }|f(x, 1)|<c(k)\left|Q_{k}\right|^{1 / p^{\prime}}
$$


it will follow that

$$
\int_{0}^{1}\left|\frac{\partial f}{\partial t}(x, t)\right| d t=\infty \quad \text { for } x \text { in } Z
$$

if we show

$$
\lim _{t \rightarrow 0} f(x, t)=\infty \quad \text { for } x \text { in } Z .
$$

But for $x$ in $Z$ we have

$$
\lim _{t \rightarrow \infty} \inf f(x, t)>\lim _{t \rightarrow \infty} \inf \left(P_{t} * f_{0}^{k} * g_{\alpha}\right)>k
$$

for all $k$. This concludes the construction of Theorem 2 .

\section{REFERENCES}

1. A. Beurling, Sur les ensembles exceptionels, Acta Math. 72 (1940), 1-13.

2. G. E. Lippman and V. L. Shapiro, Capacity and absolute Abel summability of multiple Fourier series, J. Approximation Theory 10 (1974), 313-323.

3. N. G. Meyers, A theory of capacities for potentials of functions in Lebesgue classes, Math. Scand. 26 (1970), 255-292.

Departmant of MAthematics, University of Missouri, St. LoUis, Missouri 63121 